\title{
Função Gonadal na Síndrome de Turner
}

\author{
Gonadal Function in Turner Syndrome
}

\author{
Márcia ALVES ${ }^{1}$, Margarida BASTOS ${ }^{1}$, Teresa ALMEIDA SANTOS ${ }^{2}$, Francisco CARRILHO ${ }^{1}$ \\ Acta Med Port 2013 Nov-Dec;26(6):655-663
}

\section{RESUMO}

Introdução: A síndrome de Turner caracteriza-se pela ausência, parcial ou total, de um cromossoma X no sexo feminino, sendo uma das cromossomopatias mais frequentes. O diagnóstico é realizado através do cariótipo e as suas manifestações incluem o hipogonadismo primário, antes ou após a puberdade (disgenesia gonadal). O grau de disfunção e a extensão dos defeitos gonadais são variáveis.

Objectivos: Pretendeu-se avaliar a clínica, cariótipo, função gonadal e características ecográficas do útero e ovários de mulheres com síndrome de Turner.

Material e Métodos: Estudo retrospectivo de doentes com síndrome de Turner, seguidas nos Serviços de Endocrinologia ou Reprodução Humana dos Hospitais da Universidade de Coimbra - Centro Hospitalar e Universitário de Coimbra, E.P.E. Avaliou-se toda a amostra e consideraram-se o grupo 1 (com puberdade e menarca espontânea) e grupo 2 (sem puberdade espontânea). Parâmetros avaliados: idade do estudo inicial, puberdade, cariótipo, $\mathrm{FSH}$, ecografia pélvica inicial e pós-pubertária, celioscopia e indução pubertária. Estudo estatístico: SPSS (20.0).

Resultados: Amostra: 79 doentes, $14,7 \pm 6,6$ anos. Ausência de sinais pubertários em $57,1 \%$, amenorreia primária $67,1 \%$ e secundária 6,6\%. Cariótipo: monossomia X-37,2\%, mosaico-37,2\%, alterações estruturais de X-25,6\%. Mediana da FSH 59,5mUl/mL. Ecografia inicial: útero normal-34,2\%, atrófico-65,8\%; ovários normais-21,6\%, atróficos-78,4\%, com folículos-5,1\%. Ecografia pós-pubertária: útero normal-67,9\%, atrófico-32,1\%; ovários normais-36,4\%, atróficos-63,6\%. A laparoscopia realizada em 16 (20,3\%) doentes confirmou os achados ecográficos. Duas mulheres com puberdade induzida engravidaram: uma espontaneamente, sem evolução; outra por doação de ovócitos, evolutiva. Grupo 1 (com puberdade e menarca espontânea): 20 (25,3\%) doentes, 16,1 $\pm 8,9$ anos. Puberdade na avaliação inicial: M1-22,2\%, M2-33,3\%, M3-16,7\%, M4-16,7\%, M5-11,1\%. Cariótipo: mosaico-65\%, alterações estruturais de X-20\%, monossomia X-15\%. Mediana da FSH $7 \mathrm{mUl} / \mathrm{mL}$. Ecografia inicial: útero normal-72,2\%, atrófico-27,8\%; ovários normais-63,2\%, atróficos-36,8\%. Ecografia pós-pubertária: útero normal-100\%; ovários normais-72,7\%, atróficos-27,3\%. Grupo 2 (sem puberdade

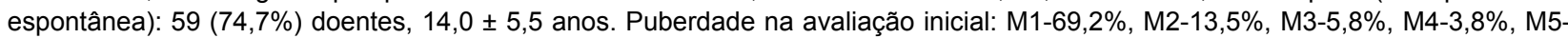
$7,7 \%$. Cariótipo: monossomia X-43,9\%, alterações estruturais de X-28,1\%, mosaico-28,1\%. Mediana da FSH $74 \mathrm{mUI} / \mathrm{mL}$. Ecografia inicial: útero normal-20,4\%, atrófico-79,6\%; ovários normais-7,4\%, atróficos-92,6\%. Ecografia pós-pubertária: útero normal-60,0\%, atrófico-40,0\%; ovários normais-27,3\%, atróficos-72,7\%. Indução pubertária aos $16,1 \pm 4,1$ anos com idade óssea $12,7 \pm 1,6$ anos. Os grupos 1 e 2 diferiram significativamente no cariótipo $(p=0,010), \mathrm{FSH}(p<0,001)$, dimensões do útero e ovários $(p<0,001)$.

Conclusões: A maioria das doentes apresentou disfunção gonadal com necessidade de indução pubertária. Ocorreu puberdade e menarca espontâneas em 25,3\% das doentes (predomínio de mosaicos). Das doentes com indução pubertária, 43,9\% apresentavam monossomia X. A fertilidade destas doentes está comprometida, podendo nalgumas situações recorrer-se a técnicas de procriação medicamente assistida para obter uma gravidez ou preservar a fertilidade.

Palavras-chave: Alterações Gonadais; Distúrbios Menstruais; Síndrome de Turner.

\section{ABSTRACT}

Introduction: Turner syndrome is characterized by the absence, total or partial, of one $\mathrm{X}$ chromosome in females, being one of the most frequent chromosomal abnormalities. Diagnosis is made by karyotype. Turner syndrome manifestations include primary hypogonadism, before or after puberty (gonadal dysgenesis). The degree and extent of gonadal disfunction are variable.

Objectives: We intended to assess clinical, karyotype, gonadal function and pelvic ultrasound characteristics in women with Turner syndrome.

Material and Methods: Retrospective study of patients with Turner syndrome followed in Endocrinology and Human Reproduction Departments of Hospitais da Universidade de Coimbra - Centro Hospitalar e Universitário de Coimbra, E.P.E. We evaluated the entire sample and considered group 1 (with spontaneous puberty and menarche) and group 2 (without spontaneous puberty). Parameters assessed: age at initial study, puberty (Tanner stages), karyotype, FSH, pelvic ultrasound (initial and after puberty), diagnostic laparoscopy and pubertal induction. Statistical Program: SPSS (20.0).

Results: Global sample: 79 patients, $14.7 \pm 6.6$ years. No pubertal signs in $57.1 \% ; 67.1 \%$ with primary amenorrhea and $6.6 \%$ with secondary amenorrhea. Karyotype: X monosomy-37.2\%, mosaicism-37.2\%, X structural changes-25.6\%. Median FSH of $59.5 \mathrm{mIU} /$ $\mathrm{mL}$. Initial ultrasound: normal uterus $34.2 \%$, atrophic uterus $65.8 \%$; normal ovaries $21.6 \%$, atrophic ovaries $78.4 \%$, ovarian follicles in 5.1\%. Post-puberty ultrasound: normal uterus $67.9 \%$, atrophic uterus $32.1 \%$; normal ovaries $36.4 \%$, atrophic ovaries $63.6 \%$. Laparoscopy was performed in $16(20.3 \%)$ patients, confirming the sonographic findings. Only two women with induced puberty became pregnant: one spontaneously, interrupted; another by donated oocytes, normal outcome. Group 1 (with spontaneous puberty and menarche): 20 (25.3\%) patients, $16.1 \pm 8.9$ years. Tanner at baseline: M1-22.2\%, M2-33.3\%, M3-16.7\%, M4-16.7\%, M5-11.1\%. Karyotype: mosaicism-65\%, X structural changes-20\%, X monosomy-15\%. Median FSH of $7 \mathrm{mUl} / \mathrm{mL}$. Initial ultrasound: normal uterus-72.2\%,

1. Serviço de Endocrinologia, Diabetes e Metabolismo. Centro Hospitalar e Universitário de Coimbra, EPE. Coimbra. Portugal.

2. Serviço de Reprodução Humana. Centro Hospitalar e Universitário de Coimbra, EPE. Coimbra. Portugal.

Recebido: 26 de Janeiro de 2013 - Aceite: 07 de Outubro de 2013 | Copyright @ Ordem dos Médicos 2013 
atrophic uterus $27.8 \%$; normal ovaries $63.2 \%$, atrophic ovaries $36.8 \%$. Post-puberty ultrasound: normal uterus $100 \%$; normal ovaries $72.7 \%$, atrophic ovaries $27.3 \%$. Group 2 (without spontaneous puberty): 59 (74.7\%) patients, $14.0 \pm 5.5$ years. Tanner at baseline: M1-69.2\%, M2-13.5\%, M3-5.8\%, M4-3.8\%, M5-7.7\%. Karyotype: X monosomy-43.9\%, X structural changes-28.1\% mosaicism-28.1\%. Median FSH of $74 \mathrm{mUI} / \mathrm{mL}$. Initial ultrasound: normal uterus $20.4 \%$, atrophic uterus $79.6 \%$; normal ovaries $7.4 \%$, atrophic ovaries 92.6\%. Post-puberty ultrasound: normal uterus $60.0 \%$, atrophic uterus $40.0 \%$; normal ovaries $27.3 \%$, atrophic ovaries $72.7 \%$. Pubertal induction at $16.1 \pm 4.1$ years, with bone age of $12.7 \pm 1.6$ years. Groups 1 and 2 differ significantly in karyotype $(p=0.010)$, median FSH $(p<0.001)$, and uterine and ovarian dimensions $(p<0.001)$.

Conclusions: Most patients had gonadal dysfunction and needed pubertal induction. Spontaneous puberty with menarche occurred in $25.3 \%$ of patients (predominantly mosaics). $43.9 \%$ of patients with pubertal induction had X monosomy. These patients fertility is compromised and, in some cases, we should refer to assisted reproductive specialist for pregnancy or fertility preservation.

Keywords: Gonadal Disorders; Menstruation Disturbances; Turner Syndrome.

\section{INTRODUÇÃO}

A síndrome de Turner (ST) caracteriza-se pela ausência, parcial ou total, de um cromossoma $X$ no sexo feminino, ${ }^{1}$ sendo uma das cromossomopatias mais frequentes. ${ }^{2}$

Ocorre em cerca de 1:2500 a 1:4000 nados-vivos do sexo feminino. ${ }^{1}$ A prevalência pré-natal é mais elevada, o que indica uma taxa maior de concepção de fetos com ST. ${ }^{2}$ No entanto, existe uma elevada mortalidade intra-uterina, especialmente durante o primeiro trimestre (com um pico na $13^{\mathrm{a}}$ semana) de gestação. ${ }^{3}$

A ST associa-se a uma enorme variedade de anomalias fenotípicas, a maioria causada por haploinsuficiência de genes normalmente expressos por ambos os cromossomas $\mathrm{X} .{ }^{4}$ Os estigmas cardinais incluem atraso de crescimento com baixa estatura, insuficiência gonadal e infertilidade. ${ }^{1,4,5}$

$\mathrm{O}$ diagnóstico é realizado através do cariótipo. Na prática clínica, cerca de metade dos doentes com ST apresenta um cariótipo $45, X$ (monossomia de $X$ ), $20 \%$ a $30 \%$ apresentam mosaicismo $(45, X$ e, pelo menos, mais uma linha celular), e os restantes têm anomalias estruturais de um cromossoma X. ${ }^{1,4}$

As manifestações de ST incluem o hipogonadismo primário, quer ocorra antes ou após a puberdade (disgenesia gonadal). O grau de disfunção e a extensão dos defeitos gonadais são variáveis. ${ }^{6}$

Durante a vida fetal, os fetos com síndrome de Turner desenvolvem um número normal de ovócitos, mas a maioria deles sofre atrésia acelerada. ${ }^{7-9} \mathrm{~A}$ causa é desconhecida, mas supõe-se que os ovócitos sem um segundo cromossoma $X$ normal não sejam viáveis. Caracteristicamente, os ovários na síndrome de Turner estão limitados a pequenas quantidades de tecido conjuntivo sem folículos ou com apenas alguns folículos atrésicos (gónadas 'em fita').

No entanto, até $30 \%$ das raparigas com ST apresentam algum grau de puberdade espontânea, cerca de $10 \%$ atinge a menarca, e estima-se que $2-5 \%$ sejam férteis. ${ }^{10-12}$ Estes números estão provavelmente subvalorizados porque muitas das mulheres com ST e com fertilidade preservada não são diagnosticadas.

A terapêutica de substituição hormonal deve começar na idade normal da puberdade, e ser continuada até aos 50 anos de idade. ${ }^{4} \mathrm{~A}$ dose de estrogénio deve ser suficiente para evitar as manifestações clínicas do hipogonadismo e as sequelas a longo prazo do défice de estrogénio, em particular, a perda de massa óssea. ${ }^{6}$
Pretendeu-se com este estudo avaliar a clínica, cariótipo, função gonadal e características ecográficas do útero e gónadas de mulheres com ST.

\section{MATERIAL E MÉTODOS}

Realizou-se um estudo retrospectivo de processos clínicos (manuscritos e informatizados) de doentes com ST, em seguimento nas consultas de Endocrinologia e/ou Ginecologia do Centro Hospitalar e Universitário de Coimbra, HUC-CHUC.

Nestes doentes avaliaram-se os seguintes parâmetros: puberdade espontânea (com ou sem menarca) ou induzida, idade inicial de avaliação em consulta, cariótipo, estádios pubertários de Tanner (à data da primeira avaliação e após puberdade), FSH pré-púbere (doseamento na primeira consulta ou antes da indução pubertária), ecografia ginecológica (inicial e após indução pubertária), realização de celioscopia e/ou cirurgia ginecológica.

Subdividiram-se as doentes em dois grupos: grupo 1 , com puberdade espontânea e menarca; e grupo 2 , sem puberdade espontânea.

Ambos os grupos foram avaliados e comparados entre si em relação a: idade de avaliação inicial em consulta, cariótipo, estádios pubertários de Tanner (à data da primeira avaliação e após a puberdade), FSH pré-púbere e ecografia ginecológica (inicial e após puberdade).

No grupo 2 foram avaliados ainda a indução pubertária, a idade cronológica e óssea à data dessa indução e o tipo de estrogénios utilizados.

O estudo estatístico foi realizado recorrendo ao programa SPSS 20.0.

\section{RESULTADOS}

Avaliaram-se 79 mulheres com diagnóstico de ST. Destas, $30(38,0 \%)$ apresentavam monossomia de X, 29 $(36,7 \%)$ eram mosaicos com cromossomas $X$ intactos e 20 $(25,3 \%)$ apresentavam alterações cromossómicas estruturais (Tabela 1).

A idade média da primeira consulta foi aos $14,7 \pm 6,6$ anos. Nessa altura, os sinais pubertários estavam ausentes em $57,1 \%$ das meninas, enquanto $18,6 \%$ apresentavam botão mamário (estádio M2), $8,6 \%$ se encontravam no estádio $\mathrm{M} 3,7,1 \%$ no estádio $\mathrm{M} 4$ e $8,6 \%$ no estádio pubertário M5; oito meninas $(10,1 \%)$ apresentavam atrofia dos genitais externos (Tabela 1).

A mediana da FSH pré-puberal foi de 59,50 (1:29,3 - 
Tabela 1 - Características da amostra total e frequências.

\begin{tabular}{|c|c|c|c|}
\hline Características da amostra & & $n$ & Percentagem (\%) \\
\hline \multirow{3}{*}{ Puberdade } & Espontânea (com menarca) & 20 & 25,3 \\
\hline & Induzida & 59 & 74,7 \\
\hline & Total & 79 & 100,0 \\
\hline \multirow{4}{*}{ Cariótipo } & Monossomia de $\mathrm{X}$ & 30 & 38,0 \\
\hline & Mosaico com X intactos & 29 & 36,7 \\
\hline & Alterações estruturais de $X$ & 20 & 25,3 \\
\hline & Total & 79 & 100,0 \\
\hline \multirow{6}{*}{ Estádio pubertário inicial* } & M 1 & 40 & 57,1 \\
\hline & M 2 & 13 & 18,6 \\
\hline & M 3 & 6 & 8,6 \\
\hline & M 4 & 5 & 7,1 \\
\hline & M 5 & 6 & 8,6 \\
\hline & Total & 70 & 100,0 \\
\hline Atrofia genitais externos & & 8 & 10,1 \\
\hline \multirow{3}{*}{ Útero pré-puberdade } & Normal & 25 & 34,2 \\
\hline & Atrófico & 48 & 65,8 \\
\hline & Total & 73 & 100,0 \\
\hline \multirow{3}{*}{ Ovários pré-puberdade } & Normais & 16 & 21,6 \\
\hline & Atróficos & 58 & 78,4 \\
\hline & Total & 74 & 100,0 \\
\hline \multirow{3}{*}{ Celioscopia - Útero } & Normal & 3 & 23,1 \\
\hline & Atrófico & 10 & 76,9 \\
\hline & Total & 13 & 100,0 \\
\hline \multirow{3}{*}{ Celioscopia - Ovários } & Normais & 1 & 7,7 \\
\hline & Atróficos & 12 & 92,3 \\
\hline & Total & 13 & 100,0 \\
\hline Gonadectomia bilateral & & 4 & 5,1 \\
\hline Histerectomia total & & 2 & 2,5 \\
\hline \multirow{6}{*}{ Estádio pubertário final* } & M 1 & 1 & 1,4 \\
\hline & M 2 & 3 & 4,3 \\
\hline & M 3 & 3 & 4,3 \\
\hline & M 4 & 15 & 21,7 \\
\hline & M 5 & 47 & 68,1 \\
\hline & Total & 69 & 100,0 \\
\hline \multirow{3}{*}{ Útero pós-puberdade } & Normal & 38 & 67,9 \\
\hline & Atrófico & 18 & 32,1 \\
\hline & Total & 56 & 100,0 \\
\hline \multirow{3}{*}{ Ovários pós-puberdade } & Normais & 20 & 36,4 \\
\hline & Atróficos & 35 & 63,6 \\
\hline & Total & 55 & 100,0 \\
\hline
\end{tabular}

*: M 1 - pré-púbere; M 2 - botão mamário; M 3 - mama e aréola maiores; M 4 - aréola e mamilo destacam-se do contorno da mama; M 5 - mama e aréola no mesmo plano; morfologia adulta.

$3: 104,8) \mathrm{mUl} / \mathrm{mL}$.

$\mathrm{Na}$ ecografia ginecológica inicial demonstrou-se útero normal em $34,2 \%$ das meninas e atrófico nas restantes $65,8 \%$; os ovários tinham aspecto normal em $21,6 \%$ dos casos, sendo atróficos em $78,4 \%$ (Tabela 1); quatro meni- nas apresentavam folículos ováricos ecograficamente visíveis.

Realizada laparoscopia diagnóstica (celioscopia) em 16 meninas com ST. A idade média de realização deste procedimento foi aos $17,1 \pm 4,9$ anos de idade; os achados 
laparoscópicos revelaram a presença de útero normal em $23,1 \%$ das meninas, atrófico nas restantes $(76,9 \%)$; os ovários revelaram-se normais em apenas uma destas meninas e atróficos em 12 (92,3\%) (Tabela 1).

Foi ainda realizada cirurgia ginecológica em seis doentes $(7,6 \%$ do total) (Tabela 1$)$, com idade média à data da cirurgia de 19,5 \pm 5,5 anos. Quatro destas doentes foram submetidas a gonadectomia bilateral por presença de material SRY em mosaicos e outras duas a histerectomia total, também por material SRY em cariótipo com mosaicismo, mas uma delas com disgerminoma do ovário adicional.

O final da puberdade, determinado por avaliação clínica ou ecográfica, ocorreu, em média aos 19,4 anos de idade. A reavaliação ecográfica de 56 mulheres revelou útero normal em $67,9 \%$, atrófico em $32,1 \%$; ovários normais em $36,4 \%$ e atróficos em $63,6 \%$ das doentes (Tabela 1).

A média de tempo de seguimento desde a primeira avaliação até ao final da puberdade foi de 6,3 \pm 3,8 anos.

Ocorreu puberdade espontânea com menarca em 20 meninas (25,3\%). Estas foram consideradas o grupo 1 ( $n=$ 20). As meninas com ST com necessidade de indução pubertária foram 59 (74,7\% do total), sendo este considerado o grupo $2(n=59)$.
No grupo 1, a maioria era portadora de cariótipo em mosaico com cromossoma $X$ intacto $(65 \%)$ existindo, no entanto, $20 \%$ com alterações estruturais cromossómicas e as restantes $15 \%$ com monossomia de $\mathrm{X}$. No grupo 2, a maioria $(45,8 \%)$ possuía monossomia de $X$, enquanto $27,1 \%$ era portadora de cariótipo com alterações estruturais cromossómicas e outros $27,1 \%$ de cariótipo em mosaico com cromossoma $\mathrm{X}$ intacto (Tabela 2).

Avaliaram-se as características dos cariótipos com alterações estruturais do cromossoma $X$ e a sua relação com os diferentes grupos (Tabela 3). Verificou-se a ocorrência de menarca espontânea em duas meninas com deleção do braço curto do cromossoma $\mathrm{X}$, em uma com mosaico com deleção do braço longo do cromossoma $X$ e noutra com mosaico com deleção do braço curto do cromossoma $X$. Não ocorreu menarca espontânea em nenhuma menina com isocromossoma ou deleção do braço longo do cromossoma $\mathrm{X}$ ou com cromossoma $\mathrm{X}$ em anel.

Comparando os grupos 1 e 2, a frequência de mosaico com isocromossoma do braço longo do $X$ foi significativamente superior no grupo $2(p=0,015)$.

Avaliaram-se ainda as diferentes linhas celulares presentes nos cariótipos em mosaico $(45, \mathrm{X}, 46, \mathrm{XX}, 47, \mathrm{XXX}$

Tabela 2 - Características dos grupos 1 (com puberdade e menarca espontâneas) e 2 (com puberdade induzida)

\begin{tabular}{|c|c|c|c|c|c|}
\hline \multirow{2}{*}{ Características } & & \multicolumn{2}{|c|}{ Grupo 1} & \multicolumn{2}{|c|}{ Grupo 2} \\
\hline & & $n$ & $\%$ & $n$ & $\%$ \\
\hline \multirow{4}{*}{ Cariótipo } & Monossomia de $\mathrm{X}$ & 3 & 15,0 & 27 & 45,8 \\
\hline & Mosaico com X intactos & 13 & 65,0 & 16 & 27,1 \\
\hline & Alterações estruturais de $X$ & 4 & 20,0 & 16 & 27,1 \\
\hline & Total & 20 & 100 & 59 & 100 \\
\hline \multirow{6}{*}{ Estádio pubertário inicial* } & M 1 & 4 & 22,2 & 36 & 69,2 \\
\hline & M 2 & 6 & 33,3 & 7 & 13,5 \\
\hline & M 3 & 3 & 16,7 & 3 & 5,8 \\
\hline & M 4 & 3 & 16,7 & 2 & 3,8 \\
\hline & M 5 & 2 & 11,1 & 4 & 7,7 \\
\hline & Total & 18 & 100,0 & 52 & 100,0 \\
\hline \multirow{3}{*}{ Ecografia inicial - Útero } & Normal & 13 & 72,2 & 11 & 20,4 \\
\hline & Atrófico & 5 & 27,8 & 43 & 79,6 \\
\hline & Total & 18 & 100,0 & 54 & 100,0 \\
\hline \multirow{3}{*}{ Ecografia inicial Ovários } & Normais & 12 & 63,2 & 4 & 7,4 \\
\hline & Atróficos & 7 & 36,8 & 50 & 92,6 \\
\hline & Total & 19 & 100 & 54 & 100,0 \\
\hline \multirow{6}{*}{ Estádio pubertário final* } & M 1 & 0 & 0,0 & 1 & 1,9 \\
\hline & M 2 & 1 & 6,2 & 2 & 3,8 \\
\hline & M 3 & 0 & 0,0 & 3 & 5,7 \\
\hline & M 4 & 1 & 6,2 & 14 & 26,4 \\
\hline & M 5 & 14 & 87,5 & 33 & 62,3 \\
\hline & Total & 16 & 100,0 & 53 & 100,0 \\
\hline \multirow{3}{*}{ Ecografia pós-puberdade Útero } & Normal & 11 & 100,0 & 27 & 60,0 \\
\hline & Atrófico & 0 & 0,0 & 18 & 40,0 \\
\hline & Total & 11 & 100,0 & 45 & 100,0 \\
\hline \multirow{3}{*}{ Ecografia pós-puberdade Ovários } & Normais & 8 & 72,7 & 12 & 27,3 \\
\hline & Atróficos & 3 & 27,3 & 32 & 72,7 \\
\hline & Total & 11 & 100,0 & 44 & 100,0 \\
\hline
\end{tabular}


e 46,XY). Não foi evidenciada diferença significativa entre os grupos relativamente à expressão das diferentes linhas celulares, apesar da linha celular $46, \mathrm{XX}$ ter sido a mais frequente nas meninas do grupo 1.

Dentro dos mosaicos com menarca espontânea (grupo 1) $92,3 \%$ possuíam linha celular $46, X X(n=12), 7,7 \%$ possuíam linha celular $47, X X X(n=1)$ e outros $7,7 \%$ possuíam linha celular $46, X Y(n=1)$.

Comparando os grupos 1 e 2 em relação à expressão quantitativa das diferentes linhas celulares presentes no sangue periférico, verificou-se uma maior expressão no grupo 1 de 46,XX e uma menor expressão das linhas 45,X, $47, X X X$ e 46,XY. Verificam-se diferenças na distribuição quantitativa das linhas celulares em mosaicos com menarca espontânea, sendo que a menarca espontânea foi mais frequentemente atingida nos mosaicos com maior expressão da linha celular $46, X X(p=0,011)$ e menos nos portadores de linhas celulares $45, X(p=0,011)$ (Tabela 4).

À data da primeira avaliação em consulta a idade média do grupo 1 era de $16,1 \pm 8,9$ anos de idade, enquanto a do grupo 2 era $14,0 \pm 5,5$ anos de idade.

Nesta altura, 22,2\% das meninas do grupo 1 apresentavam ausência de sinais pubertários (estádio M1 de Tanner), enquanto $33,3 \%$ se apresentava em estádio $\mathrm{M} 2,16,7 \%$ em estádio M3, 16,7\% em M4 e 11,1\% em M5. Na primeira avaliação do grupo 2, 69,2\% apresentavam-se sem sinais de desenvolvimento pubertário (estádio $\mathrm{M} 1$ ), 13,5\% no estádio $\mathrm{M} 2,5,8 \%$ no estádio $\mathrm{M} 3,3,8 \%$ no estádio $\mathrm{M} 4$ e $7,7 \%$ no estádio M5 de Tanner (Tabela 4).

A mediana da $\mathrm{FSH}$ pré-púbere ou à data da primeira avaliação (se já tivesse ocorrido a menarca) foi de 7 [1:53:31] $\mathrm{mUl} / \mathrm{mL}$ no grupo 1 e de 74 [1:52-3:110] $\mathrm{mUl} / \mathrm{mL}$ no grupo 2.

A avaliação ecográfica pré-pubertária no grupo 1 mostrou útero normal em $72,2 \%$ e útero atrófico em $27,8 \%$; os ovários foram visualizados como normais em $63,2 \%$, e como atróficos em $36,8 \%$. No grupo 2 evidenciou-se útero atrófico em $79,6 \%$ e útero normal em apenas $20,4 \%$; os ovários eram atróficos em $92,6 \%$ e normais em apenas 7,4\% (Tabela 4).

No grupo 2, à data da indução pubertária, a média de idade cronológica era de 16,1 $\pm 4,1$ anos e a de idade óssea de 12,7 $\pm 1,6$ anos.

Em $76,7 \%$ dos casos a puberdade foi induzida com estrogénios orais, nos restantes $23,3 \%$ com estrogénios transdérmicos. A introdução de progestativos ocorreu, em média, aos 17,2 \pm 1,8 anos de idade cronológica, com idade óssea média de 13,5 $\pm 1,1$ anos (cerca de 1,1 anos após início dos estrogénios).

Após a puberdade, ao exame objectivo, 87,5\% das muIheres do grupo 1 apresentavam-se com desenvolvimento completo (estádio M5), enquanto no grupo 2 apenas $62,3 \%$ das mulheres se encontravam no mesmo estádio (Tabela 2). Apesar de existirem diferenças entre o grupo 1 e 2, relativamente aos estádios pubertários atingidos, essas diferenças não foram significativas.

A reavaliação ecográfica do grupo 1 , no final da puberdade ou à data da última avaliação, mostrou útero normal em todas as doentes e ovários normais em $72,7 \%$, sendo atróficos em $27,3 \%$ das meninas. A reavaliação ecográfica do grupo 2 mostrou útero normal em $60,0 \%$ destas meninas, atrófico em 40,0\%; ovários normais em $27,3 \%$, atróficos em $72,7 \%$ das meninas (Tabela 4 ).

A idade média da primeira avaliação foi mais elevada

Tabela 3 - Distribuição dos cariótipos com alterações estruturais do cromossoma X.

\begin{tabular}{|c|c|c|c|c|c|c|}
\hline \multirow{2}{*}{ Alterações Estruturais do Cromossoma X } & \multicolumn{2}{|c|}{ Grupo 1} & \multicolumn{2}{|c|}{ Grupo 2} & \multicolumn{2}{|c|}{ Total } \\
\hline & $n$ & $\%$ & $n$ & $\%$ & $N$ & $\%$ \\
\hline Mosaico com isocromossoma do braço longo do $X(45, \mathrm{X} / 46, \mathrm{X}, \mathrm{i}(\mathrm{Xq}))$ & 0 & 0,0 & 11 & 55,0 & 11 & 55,0 \\
\hline Deleção do braço curto do X $(46, X, \operatorname{del}(X p))$ & 2 & 10,0 & 1 & 5,0 & 3 & 15,0 \\
\hline Isocromossoma do braço longo do $\mathrm{X}(46, \mathrm{X}, \mathrm{i}(\mathrm{Xq}))$ & 0 & 0,0 & 2 & 10,0 & 2 & 10,0 \\
\hline Mosaico com deleção do braço longo do X $(45, \mathrm{X} / 46, \mathrm{X}$, del(Xq)) & 1 & 5,0 & 0 & 0,0 & 1 & 5,0 \\
\hline Mosaico com deleção do braço curto do X $(45, \mathrm{X} / 46, \mathrm{X}$, del(Xp)) & 1 & 5,0 & 0 & 0,0 & 1 & 5,0 \\
\hline Deleção do braço longo do X $(46, X, \operatorname{del}(X q))$ & 0 & 0,0 & 1 & 5,0 & 1 & 5,0 \\
\hline Mosaico com cromossoma $X$ em anel $(45, X / 46, X, r(X))$ & 0 & 0,0 & 1 & 5,0 & 1 & 5,0 \\
\hline Total & 4 & 20,0 & 16 & 80,0 & 20 & 100,0 \\
\hline
\end{tabular}

Tabela 4 - Expressão quantitativa das diferentes linhas celulares nos cariótipos em mosaico, com cromossoma $\mathrm{X}$ intacto.

\begin{tabular}{lrr} 
Expressão quantitativa das linhas celulares nos mosaicos & $\begin{array}{c}\text { Grupo } 1 \\
(\%)\end{array}$ & $\begin{array}{r}\text { Grupo } 2 \\
(\%)\end{array}$ \\
\hline $45, X$ & $4,0[1: 1,0-3: 99,0]$ & $38,7[1: 1,0-3: 99,0]$ \\
$46, X X$ & $96,4[1: 71,3-3: 99,0]$ & $46,7[1: 0,9-3: 99]$ \\
$46, X Y$ & $1,0[1: 1,0-3: 1,0]$ & $70,0[1: 50,0-3: 90,0]$ \\
$47, X X X$ & $2,0[1: 2,0-3: 2,0]$ & $3,8[1: 0,5-3: 33,3]$ \\
\hline
\end{tabular}


nas jovens do grupo 1 (aos 16,1 \pm 8,9 anos de idade) do que nas do grupo 2, que se apresentaram em consulta mais cedo (aos 14,0 $\pm 5,5$ anos de idade). No entanto, esta diferença não se mostrou significativa no teste Mann-Whitney $(p=0,587)$.

Os grupos 1 e 2 diferiram significativamente no cariótipo $(p=0,010), \mathrm{FSH}(p<0,001)$, dimensões do útero e ovários $(p<0,001)$.

Ocorreram duas gestações, ambas em portadoras de ST com puberdade induzida (grupo 2). Uma terá sido numa mulher avaliada por amenorreia aos 33 anos, na altura com útero normal na ecografia inicial, mas com gónadas atróficas; a terapêutica hormonal nesta doente foi iniciada apenas aos 33 anos, quando foi feito o diagnóstico de ST; a gravidez terá sido espontânea aos 31 anos de idade, resultando em morte fetal in utero às 30 semanas, de acordo com informação da própria doente. A outra gestação ocorreu numa mulher com diagnóstico de ST desde os 17 anos, na altura com amenorreia primária e ausência de sinais pubertários. Por mosaicismo com cromossoma $Y$ $(45, \mathrm{X} / 46, \mathrm{XY})$ foi submetida a gonadectomia bilateral aos 18 anos de idade. Realizou fertilização in vitro com doação de ovócitos aos 40 anos, em Espanha, que resultou em gravidez gemelar, aparentemente sem complicações maternas ou fetais.

\section{DISCUSSÃO}

Nesta amostra de doentes com diagnóstico de ST, verificou-se existir relação entre função gonadal, cariótipo e níveis de gonadotropinas pré-púberes. No entanto, o facto de ser um estudo retrospectivo, através da consulta de processos clínicos, nem sempre completos em relação à informação relevante, limitou a informação e a qualidade dos resultados obtidos.

Sabe-se que o desenvolvimento pubertário espontâneo em meninas com ST deve ser avaliado pelo exame físico e doseamento hormonal da FSH, a partir dos 10 anos de idade. ${ }^{13} \mathrm{~A}$ idade média da primeira consulta no $\mathrm{CHUC}$ das doentes com ST foi aos 14,7 $\pm 6,6$ anos, mais tarde do que o recomendável. Contribuiu para esta idade média elevada o facto de se tratar de um hospital, maioritariamente, para seguimento de adultos.

A idade média com que se apresentou o grupo 1 foi aos $16,1 \pm 8,9$ anos de idade, enquanto a do grupo 2 foi aos 14,0 \pm 5,5 anos de idade. Esta diferença terá ocorrido pela presença de menarca espontânea, o que atrasou o diagnóstico de ST no grupo 1. No entanto, a diferença entre grupos não foi significativa.

O cariótipo demonstrou ser altamente preditivo da função gonadal, apesar de ter sido avaliado em linfócitos do sangue periférico, o que não terá possibilitado a detecção de algumas situações de mosaicismo de baixo grau.

Pelo teste do $\mathrm{X}^{2}$ verificou-se existir uma diferença significativa $(p=0,010)$ na distribuição dos cariótipos entre os grupos 1 e 2 (com puberdade espontânea e com puberdade induzida, respectivamente). O desenvolvimento pubertário espontâneo foi mais frequentemente observado em doentes com ST e cariótipo em mosaico, resultados consistentes com relatos anteriores. ${ }^{10,14-17}$ No entanto, alguns doentes com ST e portadores de outros cariótipos, incluindo monossomia de $\mathrm{X}$, também apresentaram puberdade espontânea com menarca, situação também já descrita. ${ }^{10,14,15,17-22}$

A correlação fenótipo/genótipo na síndrome de Turner com cariótipo em mosaico é fraca, sendo a variabilidade fenotípica dependente dos diferentes graus e padrões tecidulares de mosaicismo. Neste trabalho verificaram-se diferenças na distribuição quantitativa das linhas celulares nos cariótipos em mosaico, sendo a menarca espontânea mais frequente nos mosaicos com maior expressão da linha celular 46,XX $(p=0,011)$ e menos nos portadores de linhas celulares $45, X(p=0,011)$. Estas diferenças têm relação com o facto de existir uma linhagem de células normais $(46, X X)$.

O gene ZFX no braço curto do cromossoma X é necessário para o desenvolvimento gonadal normal. Mulheres com monossomia de $\mathrm{X}$, isocromossomo do braço longo do $X$ e deleções do braço curto, apresentam frequentemente disgenesia gonadal por haploinsuficiência deste gene. Ain$\mathrm{da}$, algumas regiões do braço longo do cromossoma $X$ relacionam-se com a falência ovárica: a falência ovárica prematura tipo 1, que abrange a região Xq26-qter, e a falência ovárica prematura tipo 2, em Xq13,3 - Xq22. Clinicamente, as doentes com anomalias destas regiões apresentam um quadro de amenorreia primária com infantilismo sexual ou de amenorreia secundária acompanhada de esterilidade. $\mathrm{Na}$ nossa amostra, as meninas com isocromossoma do braço longo do $\mathrm{X}$, mesmo nas situações de mosaicismo com outras linhas celulares, não apresentaram menarca espontânea, pertencendo ao grupo 2, o que está de acordo com o descrito anteriormente. A presença de mosaico com isocromossoma do braço longo do X na nossa amostra parece determinar uma menor razão de probabilidades de atingir a menarca de forma espontânea $(O R=0,313$; IC 95\%: $0,151-0,646)$.

Na nossa amostra apenas existe um caso com cromossoma $X$ em anel, que apresentou necessidade de indução pubertária por disgenesia gonadal.

As deleções do cromossoma $X$ na nossa amostra (do braço longo ou braço curto) apresentaram fenótipos variáveis, quase todas com menarca espontânea, excepto um caso em que houve indução pubertária. Estes resultados são contrários aos descritos na literatura em que, geralmente, as deleções do braço curto, isoladas ou em mosai$\mathrm{co}$, e as do braço longo mais distais apresentam um quadro de disgenesia gonadal. Esta diferença poderá ser devida à ausência, na nossa amostra, de deleção das regiões relacionadas com a disfunção gonadal.

À data da primeira avaliação, as jovens do grupo 1 apresentavam-se, maioritariamente, em estádios pubertários precoces (Tanner 1 em 22,2\% e Tanner 2 em 33,3\%), apesar de existir uma distribuição equilibrada entre os diferentes estádios. A grande maioria das meninas do grupo 2 encontrava-se no estádio $1(69,2 \%)$. Tal como esperado, 
esta diferença na distribuição dos estádios pubertários iniciais foi significativa ( $p=0,001)$.

As características iniciais do útero e ovários, avaliados por ecografia, diferiram entre os grupos de forma significativa no teste de Mann-Whitney $(p<0,001)$. A prevalência de útero e ovários normais foi superior no grupo 1 , concordante com a clínica.

Níveis normais ou apenas ligeiramente elevados de gonadotropinas pré-puberes, nomeadamente da $\mathrm{FSH}$, demonstram forte associação com o desenvolvimento pubertário espontâneo e a existência de folículos ováricos. ${ }^{10,12,14,17,23}$ Doseou-se a FSH antes do início da puberdade (espontânea ou induzida) ou à data da primeira avaliação (se já tivesse ocorrido a menarca) e a mediana na amostra global era de 59,50 [1:29,3 - 3:104,8] mUl/mL. No grupo 1 essa mediana foi significativamente inferior (7 [1:5-3:31] $\mathrm{mUl} / \mathrm{mL})$ à do grupo 2 (74 [1:52-3:110] $\mathrm{mUl} / \mathrm{mL})$, compatível com as diferenças pubertárias encontradas $(p<$ $0,001)$. Não foram avaliados os doseamentos de inibina $B$ e hormona anti-mulleriana, por estes não serem análise de rotina em doentes com ST e os seus resultados não estarem disponíveis na maioria dos casos.

Quando a FSH está claramente elevada, e os sinais clínicos de puberdade estão ausentes, deve ser iniciada a indução pubertária com estrogénios. O objectivo desta indução é alcançar um desenvolvimento físico e psicológico semelhante àquele que ocorre na puberdade espontânea e estabelecer um pico de massa óssea adequado. ${ }^{24}$ No entanto, como os estrogénios aceleram a fusão das epífises ósseas, o momento do seu início deve ser coordenado (frequentemente com a terapêutica com hormona de crescimento), visando alcançar o potencial máximo de crescimento, sem atrasar indevidamente o início da puberdade. ${ }^{25}$

No grupo 2, à data da indução pubertária, a média de idade cronológica era de 16,1 $\pm 4,1$ anos e a de idade óssea de 12,7 \pm 1,6 anos. Evidências recentes mostram que o início do tratamento com estrogénios aos 12 anos possibilita a evolução da puberdade, sem interferir com a altura final. ${ }^{24}$ Antes do início do tratamento deve ser realizado o doseamento de FSH para avaliar a reserva ovárica, e a ecografia pélvica para determinar o tamanho do útero e anexos.

Em $76,7 \%$ dos casos a puberdade foi induzida com estrogénios orais, nos restantes $23,3 \%$ com estrogénios transdérmicos. A via ideal de administração de estrogénios no ST é desconhecida. Para a indução pubertária em meninas sem puberdade espontânea, o regime preferencial é com estrogénios transdérmicos (preferencialmente de origem natural como o valerato de estradiol), em baixa dose, com aumento gradual da dose durante cerca de 2-3 anos, até ocorrer feminização. ${ }^{4,14}$ Os estrogénios transdérmicos têm a vantagem teórica de evitar o efeito de primeira passagem hepática, reduzindo o risco de hipertrigliceridémia e hipertensão, mas existem poucos dados comparativos. ${ }^{25}$

A introdução de progestativos ocorreu, em média, aos $17,2 \pm 1,8$ anos de idade cronológica, com idade óssea média de 13,5 $\pm 1,1$ anos (cerca de 1,1 anos após início dos estrogénios). Estes estão preconizados, em associação aos estrogénios e de forma cíclica, quando ocorrer hemorragia uterina ou após 24 meses de terapêutica com estrogénios, para prevenir a hiperplasia endometrial.

Após a indução pubertária (grupo 2) verificou-se uma evolução favorável dos órgãos reprodutores (útero e ovários), avaliados ecograficamente. A avaliação inicial mostrou um útero de tamanho normal em $20,4 \%$ dos casos, atrófico nos restantes (79,6\%). Na avaliação ecográfica final a prevalência de útero normal passou para $60,0 \%$, com apenas $40,0 \%$ das jovens apresentando útero atrófico. Neste mesmo grupo, a avaliação inicial dos ovários mostrava $92,6 \%$ atróficos. Na avaliação final, os ovários de tamanho normal passaram para $27,3 \%$ e os atróficos para $72,7 \%$. Assim, no grupo com indução pubertária, houve diferença significativa entre as avaliações ecográficas iniciais e finais, quer em relação ao tamanho do útero $(p<0,001)$, quer em relação ao tamanho dos ovários $(p=0,004)$.

Neste grupo de 79 doentes, ocorreram duas gestações, ambas em portadoras de ST com puberdade induzida (grupo 2). Uma delas terá sido espontânea e não evolutiva, numa mulher com cariótipo 45,X. Existem relatos de casos de anomalias cromossómicas na descendência e incidência aumentada de abortamentos em gravidezes espontâneas de mulheres com ST. Além disso, aquelas com anomalias estruturais de um dos cromossomas $X$ têm risco de transmitir essa anomalia às suas filhas. ${ }^{26}$

A outra gestação foi resultado de fertilização in vitro com doação de ovócitos numa mulher com mosaicismo $(45, X / 46, X Y)$, resultando em gravidez gemelar, aparentemente sem complicações maternas ou fetais. A fertilização in vitro com doação de ovócitos e a criopreservação de ovócitos ou tecido ovárico são as técnicas de reprodução assistida possíveis nestas doentes, sendo a primeira a mais frequente. ${ }^{27} \mathrm{~A}$ doação de ovócitos tem sido usada entre as mulheres com ST desde que o tratamento se tornou disponível em meados dos anos $80 .{ }^{28}$ No início, os resultados da gravidez não foram tão bons como aqueles obtidos noutras mulheres com insuficiência ovárica, ${ }^{29,30}$ mas pesquisas mais recentes demonstraram taxas de gravidez semelhantes às obtidas noutras mulheres com falência ovárica. ${ }^{31-35}$ Também as taxas de aborto foram reduzidas, provavelmente devido a uma terapêutica de substituição hormonal mais adequada, com melhor preparação do útero. As taxas de gravidez por transferência de embriões têm variado entre $30-60 \% .^{29-35}$

A hipertensão arterial é um problema em gestações por doação de ovócitos que afecta até $30 \%$ dos $\operatorname{casos}^{36,37}$ e afecta também as mulheres com ST. As mulheres com ST têm outros factores de risco além da hipertensão. A dissecção da aorta é uma complicação grave, que afecta cerca de $2 \%$ destas mulheres por ano. ${ }^{38}$ Uma gravidez pode aumentar o risco de dissecção em mulheres que têm já dilatação da aorta. Assim, é definitivamente indicado, antes de uma gravidez planeada, o diagnóstico cuidadoso desta co-morbilidade utilizando ressonância magnética. Tendo em conta estes dois factores de risco, e um provável risco aumenta- 
do de diminuição da tolerância à glicose nestas mulheres, o risco adicional de gestações gemelares não deve ser admitido. Assim, a transferência de um único embrião deverá ser um requisito.

A terapêutica de substituição hormonal adequada, preferencialmente 4 a 6 meses antes da transferência de embriões, é recomendada para optimização do útero para a gravidez. ${ }^{33,39}$

A criopreservação dos folículos no tecido cortical do ovário é amplamente aplicada em mulheres jovens pré quimio ou radioterapia, com gestações bem-sucedidas após o descongelamento do tecido e do seu transplante posterior. $^{40-42}$ Tecnicamente, obtêm-se por biópsia laparoscópica pequenos fragmentos de tecido ovárico, tentando não danificar o tecido restante para não reduzir a probabilidade de gravidez espontânea no futuro.

\section{CONCLUSÕES}

No presente estudo, a maioria das doentes com Síndrome de Turner não apresentou puberdade espontânea (74,7\% vs. $25,3 \%)$.

Comparando o grupo 1 (com puberdade espontânea)

\section{REFERENCIAS}

1. Collett-Solberg P, Gallicchio C, Coelho S, Siqueira R, Alves S, Guimarães $M$. Endocrine diseases, perspectives and care in Turner syndrome. Arq Bras Endocrinol Metab. 2011;55:550-8.

2. Gravholt C. Clinical practice in Turner syndrome. Nat Clin Pract Endocrinol Metab. 2005;1:41-52.

3. Hook E, Warburton D. The distribution of chromosomal genotypes associated with Turner's syndrome: livebirth prevalence rates and evidence for diminished fetal mortality and severity in genotypes associated with structural X abnormalities or mosaicism. Hum Genet. 1983;64:24-7.

4. Davenport M. Approach to the patient with Turner syndrome. J Clin Endocrinol Metab. 2010;95:1487-95

5. Saenger P, Wikland K, Conway G, Davenport M, Gravholt C, Hintz R, et al. Recommendations for the diagnosis and management of Turner syndrome. J Clin Endocrinol Metab. 2001;86:3061-9.

6. Saenger P. Clinical manifestations and diagnosis of Turner syndrome (gonadal dysgenesis). Uptodate [Consultado em 2012 Nov 16]. Disponível em: http://www.uptodate.com.

7. Weiss L. Additional evidence of gradual loss of germ cells in the pathogenesis of streak ovaries, J Med Genet.1971;8:540-4.

8. Singh $\mathrm{R}$, Carr $\mathrm{D}$. The anatomy and histology of $\mathrm{XO}$ human embryos and fetuses. Anat Rec. 1966;155:369-75.

9. Verlinde F. Foetal ovarian follicular reserve in Turner syndrome. In: P. Saenger, A.M. Pasquino, editors. Optimizing Health Care for Turner Patients in the 2001st Century. International Congress series no. 1212. Amsterdam: Elsevier; 2000. p.323.

10. Pasquino AM, Passeri F, Pucarelli I, Segni M, Municchi G. Italian Study Group for Turner's Syndrome. Spontaneous pubertal development in Turner's syndrome. J Clin Endocrinol Metab. 1997;82:1810-3.

11. Tarani L, Lampariello S, Raguso G, Colloridi F, Pucarelli I, Pasquino AM, et al. Pregnancy in patients with Turner's syndrome: six new cases and review of literature. Gynecol Endocrinol. 1998;12:83-7.

12. Hovatta O. Pregnancies in women with Turner's syndrome. Ann Med. 1999;31:106-10.

13. Bondy C. Turner syndrome 2008. Horm Res. 2009; 71:S52-6.

14. Alves S, Gallicchio C, Guimarães M, Santos M. Gonadotropin levels in Turner's syndrome: correlation with breast development and hormone replacement therapy. Gynecol Endocrinol. 2003;17:295-301.

15. Sybert V. Turner's syndrome. In: Cassidy SB, Allanson JE, editors. Management of genetic syndromes. $2^{\text {nd }}$ ed. Wilmington: Wiley-Liss; 2005. p.589-605.

16. Mazzanti L, Nizzoli G, Tassinari D, Bergamaschi R, Magnani C, ChiumeIlo G, et al. Spontaneous growth and pubertal development in Turner's syndrome with different karyotypes. Acta Paediatr. 1994;83:299-304.

17. Aso K, Koto S, Higuchi A, Ariyasu D, Izawa M, Igaki J, et al. Serum FSH com o grupo 2 (sem puberdade espontânea) registam-se diferenças estatisticamente significativas na distribuição dos cariótipos, nos níveis de FSH e nas dimensões do útero e ovários.

\section{AGRADECIMENTOS}

A Margarida Marques, do Serviço de Tecnologias e Sistemas de Informação do Centro Hospitalar e Universitário de Coimbra, pelo tratamento bioestatístico dos dados.

\section{CONFLITOS DE INTERESSES}

Os autores declaram a inexistência de conflitos de interesse.

\section{FONTES DE FINANCIAMENTO}

Os autores declaram a inexistência de fontes de financiamento. O presente trabalho foi previamente apresentado como comunicação oral no XIV Congresso Português de Endocrinologia e $64^{\mathrm{a}}$ Reunião Anual da SPEDM, que decorreu no Porto no Centro de Congressos do Hotel Porto Palácio, de 24 a 27 de Janeiro de 2013.

level below $10 \mathrm{mIU} / \mathrm{mL}$ at twelve years old is an index of spontaneous and cyclical menstruation in Turner syndrome. Endocr J. 2010;57:90913.

18. Gilboa Y, Rosenberg T. Typical Turner's syndrome with $45 \mathrm{XO}$ karyotype and normal menstruation. Cytogenic and histological findings. Helv Paediatr Acta. 1975;30:281-8.

19. Jacquemun $Y$, Dumon J, Buytaert P. Ovarian function in the non-mosaic Turner sundrome; a case report. Eur J Obstet Gynecol Reprod Biol. 1989;30:87-191.

20. Cools M, Rooman R, Wauters J, Jacqemyn Y, Du Caju M. A nonmosaic $45, X$ karyotype in a mother with Turner's syndrome and in her daughter. Fertil Steril. 2004;82:923-5.

21. Styne D, Grumbach M. 45,X Turner's Syndrome. In: Kronenberg HM, Melmed S, Polonsky KS, Larsen PR, editors. Williams Textbook of endocrinology. 11th ed. Philadelphia: Saunders; 2008. p.1055-8.

22. Mortensen K, Cleeman L, Hjerrild B, Nexo E, Loc H, Jeppesen EM, et al. Increased prevalence of autoimmunity in Turner syndrome-- influence of age. Clin Exp Immunol. 2009;156:205-10.

23. Borgström B, Hreinsson J, Rasmussen C, Sheikhi M, Fried G, Keros $\mathrm{V}$, et al. Fertility preservation in girls with turner syndrome: prognostic signs of the presence of ovarian follicles. J Clin Endocrinol Metab. 2009;94:74-80.

24. Cleemann L, Hjerrild B, Lauridsen A, Heickendorff L, Christiansen $\mathrm{J}$, Mosekilde L, et al. Long-term hormone replacement therapy preserves bone mineral density in Turner syndrome. Eur $\mathrm{J}$ Endocrinol. 2009;161:251-7.

25. Werther G. Australasian Paediatric Endocrine Group. Turner Syndrome Management Guidelines. [consultado em 2012 Nov 09]. Disponível em: http://www.apeg.org.au/portals/0/documents/turner_posstate.pdf

26. Verschraegen-Spae MR, Depypere H, Speleman F, Dhondt M, De Paepe A. A familial Turner syndrome. Clin Genet. 1992;41:218-20.

27. Kavoussi S, Fisseha S, Smith Y, Smith G, Christman G, Gago L. Oocyte cryopreservation in a woman with mosaic Turner syndrome: a case report. J Reprod Med. 2008;53:223-2.

28. Gutiérrez Gutierrez AM, Grimalt L, Remohí J, Pellicer A. Twin pregnancy after oocyte donation in a woman with Turner syndrome. Ginecol Obstet Mex. 1994;62:82-4

29. Rogers PA, Murphy CR, Leeton J, Hoise MJ, Beaton L. Turner's syndrome patients lack tight junctions between uterine epithelial cells. Hum Reprod. 1992;7:883-5.

30. Yaron Y, Ochshorn Y, Amit A, Yovel I, Kogosowki A, Lessing JB. Patients with Turner's syndrome may have an inherent endometrial abnormality affecting receptivity in oocyte donation. Fertil Steril. 1996;65:1249-52.

31. Press F, Shapiro HM, Cowell CA, Oliver GD. Outcome of ovum donation 
in Turner's syndrome patients. Fertil Steril. 1995;64:995-8.

32. Khastgir G, Abdalla H, Thomas A, Korea L, Latarche L, Studd J. Oocyte donation in Turner's syndrome: an analysis of the factors affecting the outcome. Hum Reprod. 1997;12:279-85.

33. Foudila T, Soderstrom-Anttila V, Hovatta O. Turner's syndrome and pregnancies after oocyte donation. Hum Reprod. 1999;14:532-5.

34. Delbaere A, Englert Y. Turner's syndrome and oocyte donation. Gynecol Obstet Fertil. 2002;30:970-8.

35. Bodri D, Vernaeve V, Figueras F, Vidal R, Guillén JJ, Coll O. Oocyte donation in patients with Turner's syndrome: a successful technique but with an accompanying high risk of hypertensive disorders during pregnancy. Hum Reprod. 2006;21:829-32.

36. Söderström-Anttila V, Tiitinen A, Foudila T, Hovatta O. Obstetric and perinatal outcome after oocyte donation: comparison with in-vitro fertilization pregnancies. Hum Reprod. 1998;13:483-90.

37. Wiggins D, Main E. Outcomes of pregnancies achieved by donor egg in vitro fertilization - a comparison with standard in vitro fertilization pregnancies. Am J Obstet Gynecol. 2005;192:2002-6.

38. Karnis MF, Zimon AE, Lalwani SI, Timmreck LS, Klipstein S, Reindollar $\mathrm{RH}$. Risk of death in pregnancy achieved through oocyte donation in patients with Turner syndrome: a national survey. Fertil Steril. 2003;80:498-501.

39. Hovatta $O$, Hreinsson JG, Fridström M, Borgström B. Fertility and pregnancy aspects in Turner syndrome. International Congress Series. 2006;1298:185-9.

40. Donnez J, Dolmans MM, Demylle D, Jadoul P, Pirard C, Squifflet J, et al. Livebirth after orthotopic transplantation of cryopreserved ovarian tissue. Lancet. 2004;364:1405-10.

41. Meirow D, Levron J, Eldar-Geva T, Hardan I, Fridman E, Zalel Y, et al. Pregnancy after transplantation of cryopreserved ovarian tissue in a patient with ovarian failure after chemotherapy. $\mathrm{N}$ Engl $\mathrm{J}$ Med. 2005;353:318-21.

42. Hovatta O. Methods for cryopreservation of human ovarian tissue. Reprod Biomed Online. 2005;10:729-34. 


\section{Função Gonadal na Síndrome de Turner \\ Acta Med Port 2013:26:655-663}

Publicado pela Acta Médica Portuguesa, a Revista Científica da Ordem dos Médicos

Av. Almirante Gago Coutinho, 151

1749-084 Lisboa, Portugal.

Tel: +351218428215

E-mail: submissao@actamedicaportuguesa.com

www.actamedicaportuguesa.com

ISSN:0870-399X | e-ISSN: 1646-0758

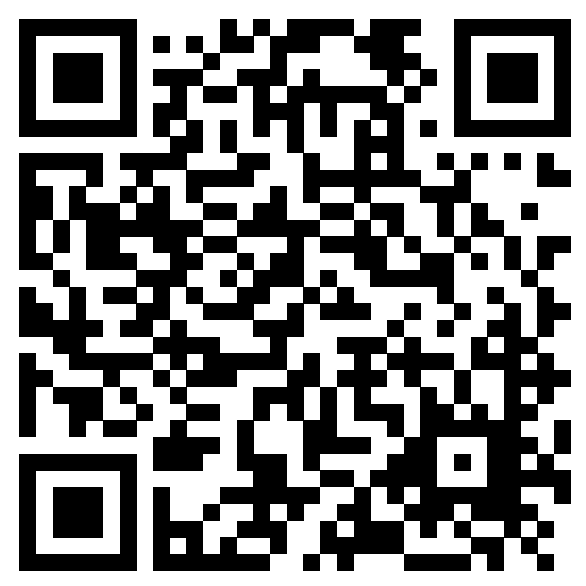

